\title{
Role of Yoga in Osteoporosis Rehabilitation
}

\author{
Donald H Lein Jr ${ }^{1 *}$, Harshvardhan Singh ${ }^{1}$ and SoJung Kim² \\ ${ }^{1}$ Department of Physical Therapy, University of Alabama at Birmingham, USA \\ ${ }^{2}$ Department of Physical Therapy, University of Massachusetts Lowell, USA
}

Submission: March 05, 2018; Published: April 30, 2018

*Corresponding author: Donald H Lein Jr, Department of Physical Therapy, University of Alabama at Birmingham, SHPB 376, $17202^{\text {nd }}$ Avenue South, Birmingham, AL, 35294-1212, USA, Tel: 205-934-0241; Fax: 205-975-7787; Email: dlein@uab.edu

\begin{abstract}
There is a lot of interest among clinicians and clients/patients concerning the utility of Yoga to build bone mineral density and decrease the risk of osteoporosis-related fractures. By examining the previous literature, our present review offers recommendations concerning the utility of Yoga for the prevention and management of osteoporosis and its related fractures. There is conflicting evidence that Yoga can help build bone mineral density (BMD). The mixed findings regarding the effects of yoga on bone could be due to different types of Yoga being used in research studies that are not performed at a moderate or high intensity as recommended by the American College of Sports Medicine to positively affect bone. Many forms of Yoga also do not generate enough ground reaction force to positively improve bone health. Yoga, however, has been shown to improve balance which may potentially prevent falls and thus osteoporosis-related fractures associated with falls. Thus Yoga could be integrated into physical therapy care. However, decisions to include Yoga should only be made knowing the clients/patients dual energy $\mathrm{x}$-ray absorptiometry central scores and medical backgrounds. Once a patient is determined to be medically fit for doing Yoga, physical therapists should incorporate Yoga exercises that are safe, build bone, and improve balance. This review article will provide some guidelines for clinicians to successfully integrate Yoga exercise into osteoporosis care.
\end{abstract}

Keywords: Bone health; Exercise; Balance; Prevention

Abbreviations: ACSM: American College of Sports Medicine; BMD: Bone Mineral Density; DXA: Dual Energy x-ray Absorptiometry; METS: Metabolic Equivalents; NOF: National Osteoporosis Foundation

\section{Introduction}

Different national and international organizations [1-3] define osteoporosis in various ways with the primary idea that it is a metabolic disorder that decreases bone strength which increases fracture risk. In the United States (US), roughly 2 million osteoporosis fractures occur per year leading to significantly higher national costs associated with osteoporosisrelated fractures [4]. Osteoporotic fractures lead to increased risk of morbidity and mortality [4]. Burge et al. [5] estimated that osteoporosis-related fractures cost 17 billion dollars in the US in 2005 which is projected to increase to 25.3 billion dollars in 2025 .

Per the mechanostat model, muscles are the chief agents for producing mechanical load which in turn stimulates osteogenesis and thus leading to increased bone density and bone strength [6]. There is evidence that bone mineral density (BMD) is lower in older individuals with lower muscle strength [7-9]. Thus, it is recommended that older men and postmenopausal women aged 50 years or more should perform "regular weightbearing and muscle-strengthening exercise to improve agility, strength, posture, and balance; maintain or improve bone strength, and reduce the risk of falls and fractures [10]." This is in line with the American College of Sports Medicine (ACSM) recommendation of performing weight-bearing and/or resistive

exercises throughout a person's lifespan to decrease the risk of osteoporosis and its related fractures [11]. According to the ACSM, the prescribed intensity, frequency, and duration of these exercises differ with age but are generally performed with moderate to high intensity in terms of bone-loading forces, on most days of the week, and each bout of exercises should last 20 to 60 minutes [11]. The ACSM also suggested that elderly men and women should follow the exercise recommendations for adults to combat BMD loss but health professionals should also include balance activities to help prevent falls due to ageingassociated deficits in balance [11].

One type of exercise that is gaining popularity in the US and Europe is Yoga. Cramer et al. [12] reported that 21 million Americans practiced Yoga in the past 12 months in a national survey distributed in 2012 and that two thirds of responding clinicians recommended Yoga to improve their clients/patients health status. These authors also found that the respondents attended Yoga to improve wellness or prevent disease $(78.4 \%)$, increase energy (66.1\%) or to improve immune function (49.7\%). The survey respondents also practiced Yoga to address medical conditions such as back pain (19.7\%), stress (6.4\%) and arthritis 
(6.4\%). This data emphasizes that Yoga is liked by clients/ patients and has a high degree of acceptability by clinicians. Physical therapists (PTs) are often asked by their clients/patients about the usefulness of Yoga to help improve bone health and reduce the risk of fractures related to osteoporosis. Our current mini-review explores this question by examining the previous literature and provides recommendations concerning the integration of Yoga for the prevention and care of osteoporosis and its related fractures. Our main aim is to specifically review the effects of Yoga on bone health.

\section{Discussion}

Previous investigators have studied many different styles of Yoga which can complicate the recommendations that can be drawn from them. In a recent systematic review of 306 randomized control trials, the authors identified 52 different Yoga styles [13]. The most common Yoga styles were hatha Yoga (36 studies), lyengar Yoga (31 studies), pranayama Yoga (26 studies) and integrated Yoga approaches. (15 studies). Despite the large variety of Yoga styles, the authors found that 91-percent of these randomized trials (277 studies) resulted in positive health outcomes and that the type of Yoga practiced was not statistically significantly related to a positive health outcome. Yoga has been shown to have positive effects on various health and quality of life measures as well as medical conditions [14]. These findings also include health measures that PTs would address with a client/patient to prevent or rehabilitate osteoporosis such as rehabilitation of muscle strength, range of motion/flexibility, balance, and bone strength.

While the practice of Yoga may result in many health benefits including a sense of well-being and accomplishment [14], the intensity of Yoga may not satisfy the recommendations by the ACSM [15] detailed earlier [11]. Meyer [16] performed a systematic review of metabolic intensity of Yoga. She found that the majority of postures and full Yoga session exercises were classified as lightaerobic intensity ( $<3$ METs) and a small minority of exercises were classified as moderate aerobic intensity (3-6 METs). Intensities for individual postures averaged 2.2 METs while individual breathing control exercises averaged 1.3 METs. This data suggested that Yoga should be typically classified as light intensity physical activity and would not adequately affect bone health for healthy individuals according to ACSM guidelines for healthy bone. Furthermore, Wilcox and colleagues (2012) found that a sequence of 28 Hatha Yoga postures produced low peak ground reaction forces to upper and lower extremities that ranged from 0.22 to 1.47 times body weight [17]. Notably, ground reaction forces of 2 times the body weight are needed to positively affect bone health [18]. In Yoga, Sun Salutations (SS) are used as a yoga sequence, where yoga postures are performed dynamically and aerobically with combinations of forward and backward bending postures. Previous studies reported that SS sequence theoretically produced dynamic joint strains and moments similar to other daily activities and exercises
[19], improved lower body muscle strength [20] and reached intensities to potentially impact bone health [15]. Although yoga has the potential to be an alternative physical activity to improve bone health, there is a lack of high quality evidence for this type of intervention.

Investigators have reported that Yoga affects bone turnover markers but the results differ. These differences may be due to the majority of Yoga styles not meeting ACSM intensity recommendations to promote bone health [16]. Phoosuwan et al. [21] reported that a 12-week weight-bearing Yoga training program performed 3 days a week by postmenopausal women with normal BMD, which was determined via heel ultrasound, significantly decreased the bone resorption marker in the Yoga group compared to the control group. They also found no differences between the two groups in terms of the bone formation marker which decreased from baseline to post intervention. In contrast, a 12-week, 1 day a week Yoga training study with postmenopausal women with osteopenia showed a moderate correlation between the amount of Yoga practiced and a bone formation marker but not with the bone resorption marker [22], suggesting yoga may in part produce an anabolic stimulus on bone. Reasons for these differences other than intensity of the Yoga program, may also be due to diurnal variation and food intake which can affect metabolic bone marker measurements. In a RCT that controlled for these factors, the investigators studied a 2-day per week, high intensity Yoga program (power yoga) of 8-month duration, and reported that the bone formation marker did not change in healthy premenopausal women (35-50 years) that completed the intervention but the control group had a significant reduction in this bone marker [23]. Finally, no change was found in either group in regard to the bone resorption marker. While gaps of knowledge exist concerning Yoga's effect on bone turnover markers, it appears that Yoga promotes bone formation in adults who have normal bone density or diagnosed with osteopenia -although the underlying mechanism is still unclear. To our knowledge, there is no data on effects of Yoga on bone health in children and adolescents, and individuals with osteoporosis. Thus, future studies are needed to investigate its overall impact on bone turnover markers across both sexes and all age groups.

Using RCT designs, several group of investigators studied the effect of Yoga on various bone health measures. Investigators of one study found that after either a 6-month low-intensity Yoga program or progressive resistance exercises in older women, bone mineral content (BMC) did not change in either group [24]. Another investigative group reported that BMC increased in healthy older men after 14 months of either an aerobic exercise program or light exercise program, but BMC, measured with single photon absorptiometry, did not increase in the women or men who performed non-aerobic or light intensity yoga [25]. Investigators in a more recent RCT showed no group, time, or interaction effect when comparing areal BMD, bone strength, 
and volumetric BMD changes in healthy postmenopausal women who performed a high intensity Yoga program 2 days a week to a control group after 8 months [23]. Investigators of two different experimental pre-post group studies, reported increased BMD after Yoga interventions [26,27]. In one study, men and women with different bone BMD classifications performed a 12-minute daily Yoga regimen for 2 years [26] while in the other study postmenopausal women with osteoporosis completed a 1-hour Yoga session, 4 days a week, for 6 months [27]. The results of these studies need to be interpreted with caution due to biases inherent to no randomization and lack of a control group. In short, more Yoga studies need to be performed that follow ACSM guidelines for exercise prescription to promote positive bone health to determine if moderate to high intensity Yoga improves bone health. Quantifying yoga session intensity, including weightbearing yoga postures and SS is urgently needed to provide an evidence-based optimal yoga program for bone health.

While improving BMD decreases fracture risk [10], improving balance will also decrease fall risk which in turn has potential to prevent osteoporosis-related fractures [10]. Two groups of investigators performed systematic reviews and found that Yoga can also improve balance in individuals $[28,29]$. Youkana et al. [28] performed a systematic review and meta-analysis found that Yoga interventions had a small positive effect on balance in adults aged 60 years or greater. Another systematic review found that 11 out of 15 studies found that Yoga interventions yielded positive results on at least one balance measure in a range of ages (10 to 90 years) [29].

\section{Conclusion}

Taken together, Yoga, if performed at dynamic moderate to high levels of intensity, may positively affect BMD and balance at all ages. Better BMD and balance can potentially decrease the risk of osteoporosis-related fractures and the associated morbidity and mortality. Yoga also has a potential to be incorporated in into PT practice; however, more evidence is required for the inclusion of yoga in clinical practice and guidelines. In a previous systematic review, Smith \& Boser [30] stated that clinicians should not use a one size fits all approach when working with individuals interested in Yoga. Furthermore, researchers of another systematic review cautioned that patients with compromised bone, like osteoporosis, should avoid forceful or competitive yoga practices [31]. We recommend that prior to starting a yoga routine with clients/patients that clinicians ensure that they do not have osteoporosis based on central dualenergy X-ray absorptiometry (DXA) scans using World Health Organization guidelines. The following recommendations are based on the DXA results [32].

If a patient/client has a T-score of greater than (-) 2.5 and no history of a fracture after the age of 50 years then intense Yoga could be prescribed as outlined by the ACSM [9]. Based on medical history and individual-specific needs, PTs should explore the potential of high impact weight bearing activities including high intensity weight-bearing Yoga sequences or high intensity resistance training if changes in BMD is going to occur [11].

Clients/patients who have spinal osteoporosis ((T-score $<(-) 2.5)$ with or without vertebral fractures should avoid poses that increase trunk flexion and trunk rotation. Those clients/patients with hip osteoporosis should avoid deep hip stretches. For safety, individuals with osteoporosis should not allow instructors to perform overpressures with them. These recommendations are consistent with National Osteoporosis Foundation (NOF) guidelines [33]. The NOF provides poses (e.g., that avoid rounding poses, deep twists, deep hip stretches, warrior I) that can be safely performed by individuals with osteoporosis, but we recommend that PTs should supervise these Yoga styles to make sure that they are performed in a safe manner. Finally, all older adults or individuals with medical conditions should be screened for balance to insure safety with any Yoga style. We recommend that clinicians used The Centers of Disease Control and Prevention STEADI (Stopping Elderly Accidents, Deaths \& Injuries) tool kit to screen and assess balance [34]. If balance issues exist, PTs should design a balance program and modify a Yoga program to improve balance prior to clients/patients independently performing Yoga poses at home.

\section{Conflicts of Interest}

The authors certify that they have no affiliations with or financial involvement in any organization or entity with a direct financial interest in the subject matter or materials discussed in the article.

\section{References}

1. NIH Consensus Development Panel on Osteoporosis Prevention, Diagnosis, and Therapy (2001) Osteoporosis prevention, diagnosis, and therapy. JAMA 285(6): 785-795.

2. Lewiecki EM, Compston JE, Miller PD, Adachi JD, Adams JE, et al. (2011) Official positions for FRAX® bone mineral density and FRAX ${ }^{\circledR}$ simplification from Joint Official Positions Development Conference of the International Society for Clinical Densitometry and International Osteoporosis Foundation on FRAX®. J Clin Densitom 14(3): 226-236.

3. Siris ES, Adler R, Bilezikian J, Bolognese M, Dawson Hughes B, et al. (2014) The clinical diagnosis of osteoporosis: a position statement from the national Bone Health Alliance Working Group. Osteoporos Int 25(5): 1439-1443.

4. National Osteoporosis Foundation (2018) Osteoporosis fast facts.

5. Burge R, Dawson Hughes B, Solomon DH, Wong JB, King A, et al. (2007) Incidence and economic burden of osteoporosis-related fractures in the United States, 2005-2025. J Bone Min Res 22(3): 465-475.

6. Frost HM (2003) Bones Mechanostat: A 2003 Update. Anat Rec ADiscov Mol Cell Evol Biol 275(2): 1081-1101.

7. Singh H, Kim D, Bemben MG, Bemben DA (2017) Relationship between muscle performance and DXA-derived bone parametrs in communitydwelling older adults. J Musculoskelet Neuronal Interact 17(2): 50-58.

8. Tachiki T, Kouda K, Dongmei N, Tamaki J, Iki M, et al. (2017) Muscle strength is associated with bone health independently of muscle mass in postmenopausal women: the Japanese population-based osteoporosis study. J Bone Miner Metab pp. 1-7. 


\section{Journal of Yoga and Physiotherapy}

9. Li YZ, Zhuang HF, Cai SQ, Lin CK, Wang PW, et al. (2018) Low grip strength is a strong risk factor of osteoporosis in postmenopausal women. Orthp Surg 10(1): 17-22.

10. Cosman F, De Beur SJ, LeBoff MS, Lewieki EM, Tanner B, et al. (2014) Clinician guide to prevention and treatment of osteoporosis. Osteoporos Int 25(10): 2359-2381.

11. Korht WM, Bloomfield SA, Little KD, Nelson ME, Yingling VR (2004) American College of Sports Medicine Physical activity and bone health Med Sci Sports Exerc 36(11): 1985-1996.

12. Cramer H, Ward L, Steel A, Lauche R, Dobos G, et al. (2016) Prevalence, patterns, and predictors of Yoga use. Results of a U.S. nationally representative survey. Am J Prev Med 50(2): 230-235.

13. Cramer H, Lauche R, Langhorst J, Dubos G (2016) Is one Yoga style better than another? A systematic review of associations of Yoga style and conclusions in randomized Yoga trials. Complement Ther Med 25 178-187.

14. Field T (2016) Yoga research review. Complement Ther Clin Pract 24: 145-161.

15. Hagins M, Moore W, Rundle A (2007) Does practicing hatha yoga satisfy recommendations for intensity of physical activity which improves and maintains health and cardiovascular fitness? BMC Complement Altern Med 7: 40.

16. Larson Meyer DE (2016) A systematic review of the energy cost and metabolic intensity of Yoga. Med Sci Sports Exerc 48(8): 1558-1569.

17. Wilcox SJ, Hagar R, Lockhart B, Seeley MK (2012) Ground reaction force generated by twenty-eight Hatha Yoga postures. Int J of Exerc Sci 5(2): 114-126.

18. Grove KA, Londeree BR (1992) Bone density in postmenopausal: high impact vs low impact exercise. Med Sci Sports Exerc 24(11): 11901194.

19. Omkar SN, Mour M, Das D (2011) A mathematical model of effects on specific joints during practice of the Sun Salutation -a sequence of yoga posture. J Bodyw Mov Ther 15(2): 201-208.

20. Kim S, Bemben MG, Bemben DA (2012) Effects of an 8-month yoga intervention on arterial compliance and muscle strength in premenopausal women. J Sports Sci and Med 11(2): 322-330.

21. Phoosuwan M, Kritpet T, Yuktanandana P (2009) The effects of weight bearing yoga training on the bone resorption markers of the postmenopausal women. J Med Assoc Thai (92 Supppl 5): S102-S108.
22. Balk J, Gluck M, Bernado L, Catov J (2009) The effect of Yoga on markers of bone turnover in osteopenic women: a pilot study. Int J Yoga Therap 19(1): 63-68.

23. Kim S, Bemben MG, Knehans AW, Benbem DA (2015) Effects of an 8-month Ashtanga-based Yoga intervention on bone metabolism in middle-aged premenopausal women: a randomized controlled study. J Sports Sci Med 14(4): 756-768.

24. Ades PA, Savage PD, Brocha M, Tischer MD, Lee NM, et al. (2005) Resistance training increases total daily energy expenditure in disabled older women with coronary heart disease. J Appl Physiol 98(4): 1280-1285.

25. Blumenthal JA, Emery CF, Madden DJ Schniebolk S, Riddle MW, et al. (1991) Effects of exercise training on bone density in older men and women. J Amer Geriatr Soc 39(11): 1065-1070.

26. Lu YH, Rosner B, Chang G, Fishman LM (2016) Twelve-minute daily Yoga regimen reverses osteoporotic bone loss. Top Geriatr Rehabil 32(2): 81-87.

27. Motorwala ZS, Kolke S, Panchai PY, Bedekar NS, Sancheti PK, et al (2016) Effects of Yogasanas on osteoporosis in postmenopausal women. Int J Yoga 9(1): 44-48.

28. Youkhana S, Dean CM, Wolff M, Sherrington C, Tiedemann A (2016) Yoga-based exercises improves balance and mobility in people aged 60 and over: a systematic review and meta-analysis. Age Aging 45(1): 21-29.

29. Jeter PE, Nkodo AF, Moonaz SH, Dagnelie G (2014) A systematic review of Yoga for balance in a healthy population. J Altern Complement Med 20(4): 221-232.

30. Smith EN, Boser A (2013) Yoga, vertebral fractures, and osteoporosis: research and recommendations. Int J Yoga Therap 23(1): 17-23.

31. Cramer H, Krucoff C, Dobos G (2013) Adverse events associated with yoga: a systematic review of published case reports and case series. PLoS ONE 8(10): e75515.

32. World Health Organization (1994) Assessment of fracture risk and its application to screening for postmenopausal osteoporosis. Report of a WHO study group. World Health Organ Tech Rep Ser 843: 1-129.

33. National Osteoporosis Foundation (2016) So you want to do Yoga? and you have Osteoporosis.

34. Centers for Disease Control and Prevention (2017) STEADI- older adult prevention.

\section{Your next submission with Juniper Publishers will reacsh you the below assets}

- Quality Editorial service

- Swift Peer Review

- Reprints availability

- E-prints Service

- Manuscript Podcast for convenient understanding

- Global attainment for your research

- Manuscript accessibility in different formats

( Pdf, E-pub, Full Text, Audio)

- Unceasing customer service

Track the below URL for one-step submission https://juniperpublishers.com/online-submission.php 